\title{
CONTINUOUS SURJECTIONS FROM CANTOR SETS TO COMPACT METRIC SPACES
}

\section{ALAN H. SCHOENFELD ${ }^{1}$}

ABSTRACT. We present here a new proof of the fact that any compact metric space is the continuous image of the Cantor discontinumm.

It is well known that if $C$ is the Cantor "middle thirds" set and $X$ a nonempty compact metric space, there is a continuous surjection $f: C \rightarrow X$. The two "standard" proofs of this are given in the references below. We present here a third, more direct proof. In view of the fact that any compact, perfect, totally disconnected, metrizable space (called a Cantor set) is homeomorphic to $C$, it is sufficient to prove the following

Theorem. Let $X$ be a nonempty compact metric space. Then there is a Cantor set $K$ and a continuous surjection $g: K \rightarrow X$.

Proof. By an easy inductive argument based on the compactness of $X$, we can obtain a sequence $\left\{N_{n}: n=1,2, \cdots\right\}$ of integers greater than 1 and a sequence

$$
\mathcal{U}_{n}=\left\{U_{i_{1}, i_{2}, \cdots, i_{n}}: 1 \leq i_{j} \leq N_{j} \text { for } 1 \leq j \leq n\right\}
$$

of covers of $X$ such that for each $n$

(1) each $U \in \mathcal{U}_{n}$ is the closure of a nonempty open set in $X$, of diameter less than $2^{-n}$;

(2) for fixed $i_{1}, i_{2}, \cdots, i_{n}$,

$$
U_{i_{1}, i_{2}, \cdots, i_{n}}=\bigcup\left\{U_{i_{1}, i_{2}, \cdots, i_{n}, i_{n+1}} \in \mathcal{U}_{n+1}\right\} .
$$

Now for each $n=1,2, \cdots$ let $S_{n}$ be the discrete topological space consisting of the points $1,2, \cdots, N_{n}$. Set $K=X_{n=1}^{\infty} S_{n}$, with the product topology. One verifies trivially that $K$ is a Cantor set.

Now define the map $g: K \rightarrow X$ by

$$
g(\langle i\rangle)=g\left(\left\langle i_{1}, i_{2}, \cdots\right\rangle\right)=U_{i_{1}} \cap U_{i_{1}, i_{2}} \cap U_{i_{1, i_{2}, i_{3}}} \cap \cdots
$$

Received by the editors March 5, 1973.

AMS (MOS) subject classifications (1970). Primary 54C05, 54G05.

1 Supported by NSF grant GP 28580. 
We have by (1), (2), and the Cantor intersection theorem that $g$ is well defined. $g$ is clearly a surjection, and we now show it to be continuous. For let $x=g(\langle i\rangle)$ as defined in (3), and $V$ be any neighborhood of $x$. We will produce a neighborhood $U$ of $\langle i\rangle$ with $g(U) \subset V$. Since $V$ is open and the diameters of the $U_{n}$ decrease uniformly to zero, we have that there is some $N$ such that $\hat{U}=U_{i_{1}, i_{2}, \cdots, i_{N}}$ is contained in $V$. Consider now the set $U=\left\{i_{1}\right\} \times\left\{i_{2}\right\} \times \cdots \times\left\{i_{N}\right\} \times X_{n=N+1}^{\infty} S_{n}$. U is obviously a neighborhood of $\langle i\rangle$; and $g(U)=\hat{U} \subset V$, completing the proof.

\section{REFERENCES}

1. John L. Kelley, General topology, Van Nostrand, Princeton, N. J., 1955, pp. 165-166. MR 16, 1136.

2. John G. Hocking and Gail S. Young, Topology, Addison-Wesley, Reading, Mass., 1961, pp. 126-128. MR 23 \#A2857. 94305

DEPARTMENT OF MATHEMATICS, STANFORD UNIVERSITY, STANFORD, CALIFORNIA

Current address: Department of Mathematics, University of California, Davis, California 95616 